\title{
Asociación entre legitimidad organizacional y desempeño organizacional. Caso: Escuela administración de empresas
}

\author{
Association between organizational \\ legitimacy and organizational performance. \\ Case: School Business Administration \\ DOI: http://dx.doi.org/10.17981/econcuc.39.2.2018.10
}

Artículo de investigación. Fecha de recepción: 05/06/2018 Fecha de aceptación: 20/12/2018

\author{
Dora Esther Fonseca Pinto \\ Universidad Pedagógica y Tecnológica de Colombia-UPTC (Colombia) \\ fonesther@gmail.com \\ Jenny Mairena Herrera Rodríguez \\ Universidad Pedagógica y Tecnológica de Colombia-UPTC (Colombia) \\ jmairena.herrera@gmail.com
}

Para citar este artículo:

Fonseca, D. y Herrera, J. (2018). Asociación entre legitimidad organizacional y desempeño organizacional. Caso: Escuela administración de empresas. Económicas CUC, 39(2). 159-170. DOI: http://dx.doi.org/10.17981/econcuc.39.2.2018.10

\section{Resumen:}

El presente artículo se deriva del proyecto de investigación "Asociación entre legitimidad organizacional y desempeño organizacional, caso Escuela de Administración de Empresas", cuyo objetivo es dar a conocer los resultados obtenidos en la ejecución del proyecto, el cual se trabajó bajo una metodología cualitativa usando el método estudio de caso bajo la técnica de la aplicación de entrevistas a los actores y revisión bibliográfica para de esta manera comprender e interpretar el grado de legitimidad de la Escuela y su desempeño organizacional. Se pudo confrontar teóricamente la relación de los conceptos en primera instancia, para luego ratificarla en el estudio práctico.

Palabras clave: Legitimidad organizacional, desempeño organizacional, teoría institucional, estudio de caso.

\section{Abstract:}

This article is derived from the research project "Association between organizational legitimacy and organizational performance, case School of Business Administration", whose objective is to publicize the results obtained in the execution of the project, which was worked under a qualitative methodology using the case study method under the technique of the application of interviews to the actors and bibliographic review in order to understand and interpret the degree of legitimacy of the School and its organizational performance. It was possible to theoretically confront the relationship of the concepts in the first instance, and then ratify it in the practical study.

Keywords: Organizational legitimacy, organizational performance, institutional theory, case study. 


\section{Introducción}

Las organizaciones buscan diferentes mecanismos para lograr sobrevivir y mantenerse en el tiempo, indagando en su legitimación para poder institucionalizarse (DiMaggio \& Powell, 1983; Zucker, 1987). En las últimas décadas este es un tema que ha cobrado gran interés entre los académicos y directivos de las empresas (Deephouse y Suchman, 2008), profundizando cada vez más en él, desarrollando investigaciones relacionadas con esta cuestión, para lo cual se ha convertido en uno de los elementos fundamentales para entender la teoría institucional, es así, como para Bansal y Clelland (2004), la búsqueda de la legitimidad en el contexto institucional viene siendo uno de los principales argumentos en el momento de explicar el comportamiento de organizaciones hacia la sostenibilidad.

Los pioneros en estudiar la teoría institucional han considerado que las organizaciones han logrado su legitimidad cumpliendo las normas, creencias y reglas generales (Meyer \& Rowan, 1977; DiMaggio \& Powell, 1983; Scott, 1995). Meyer \& Rowan (1977) sugieren que tanto la eficiencia técnica como la conformidad con los mitos institucionales pueden ser precursores de la legitimidad, en tanto las organizaciones los empleen para generar estrategias de mejora.

La importancia del estudio de la legitimidad es presentado por Meyer \& Rowan (1977) como un factor clave para la supervivencia de las organizaciones; de igual manera se puede concebir como una componente de gran importancia para determinar el éxito o el fracaso de las organizaciones (Díez, Blanco y Prado, 2010; Arnold, Handelman y Tigert, 1996). Los estudios de Alcantara, Mitsuhashi y Hoshino (2006) y Tornikoski \& Newbert (2007) demostraron la existencia de una relación directa y positiva entre las acciones estratégicas dirigidas a ganar legitimidad y el éxito organizativo. Realizando este tipo de estudios también se encuentran autores como Reimann, Ehrgott, Kaufmann y Carter (2011), quienes enmarcan su investigación en el estudio de la relación entre legitimidad local y desempeño de los empleados, al igual que Jun, Qiuzhen y Qingguo (2011) que buscan en igual forma la relación directa entre legitimidad interna y desempeño organizacional.

Para la presente investigación se estudió la asociación entre legitimidad y desempeño organizacional, teniendo en cuenta que un factor conlleva al otro; se desarrolló bajo el método estudio de caso, con el programa de Administración de Empresas de la Universidad Pedagógica y Tecnológica de Colombia, en la Sede Seccional Sogamoso. Teniendo en cuenta que los autores del estudio pertenecen a esta escuela y buscan generar un aporte investigativo que contribuya al beneficio de la institución, los resultados obtenidos serán de aplicación académica.

La gestión de las organizaciones implica un buen aprovechamiento de los recursos para el logro de los objetivos y la mejora del desempeño. La teoría institucional enmarca el estudio de las organizaciones en el ámbito social, cultural e histórico en su interacción con el entorno para sobrevivir y legitimar su actuación y existencia. Según (Suchman, 1995; Hunt \& Aldrich, 1996; Zimmerman y Zeitz, 2002) señalan la legitimidad como un factor importante a tener en cuenta cuando se busca el éxito de las organizaciones; la teoría institucional subraya que la supervivencia y el éxito organizativo dependen de la legitimidad (Meyer \& Rowan, 1977), y con base en lo anterior se puede afirmar que la legitimidad es clave para cualquier tipo de organización.

Autores como Suchman (1995) define la legitimidad como "la percepción generalizada o asunción de que las actividades de una entidad son deseables, correctas o apropiadas dentro de algún sistema socialmente 
construido de normas, valores, creencias y definiciones". Por otro lado, se considera por legitimidad a la relación lógica entre los valores, normas y expectativas de la sociedad con las actividades y los resultados de la organización (Dowling \& Pfeffer, 1975; Ashforth \& Gibbs, 1990) en (Díez, Blanco y Prado, 2010). Es así, como las organizaciones pueden medir la dificultad y la eficacia de los esfuerzos de legitimación de los objetivos propuestos por la misma.

Dentro del esfuerzo por profundizar en el tema, algunos investigadores han intentado calcular la legitimidad usando varias medidas indirectas o aproximaciones relacionadas con las fuentes de legitimidad (DiMaggio \& Powell, 1983; Aldrich \& Fiol, 1994; Scott, 1995; Suchman, 1995; Hunt \& Aldrich, 1996). Fombrun \& Shanley (1990), también han basado la medición a través de cuestionarios con expertos, al ser estos los que permiten justificar y soportar los estudios; del mismo modo Human \& Provan (2000), Rutheerford \& Buller (2007) y Low \& Johnston (2008) han utilizado entrevistas semi estructuradas para este fin.

Suchman (1995) presenta los tipos de legitimidad como son la legitimidad pragmática, moral y cognitiva, las cuales serían las piedras angulares a la hora de caracterizar y/o medir dicho constructo. Otros autores han realizado planteamientos similares, como el argumentado por Scott (1995), quien considera tres fuentes de legitimidad a las que llamó reguladora, normativa y cognitiva. Y Hunt \& Aldrich (1996) también consideran tres fuentes de legitimidad: sociopolítica reguladora, sociopolítica normativa y sociopolítica cognitiva. Por último, Zimmerman y Zeitz (2002) añaden una nueva fuente de legitimidad determinada por el sector donde opera la organización. Los tipos de legitimidad como pragmático, moral y cognitivo se darán cuando las actividades de una organización son aceptadas y aprobadas como correctas por la sociedad según la estructura normativa que se maneje (Díez, Blanco y Prado, 2010)

Por su parte, uno de los autores que mejor define la legitimidad cognitiva es (Scott, 1994), quien afirma que esta dependerá de profesionales y científicos especializados que aprueben la actuación de las actividades que se realizan a diario en una organización. Mientras tanto, el tipo de legitimidad pragmática tiene también que ver con sus grupos de interés y la relación que entre estos pueda surgir, es decir el grado de influencia que puedan llegar a generar (Suchman, 1995).

Otras investigaciones han demostrado que la percepción de legitimidad modera los efectos del poder sobre la aproximación conductual (Lammers, Galinsky, Gordijn y Otten, 2008), la persistencia (Willis, Guinote y Rodríguez-Bailón, 2010), el optimismo (Willis y Rodríguez-Bailón, 2011) y la creatividad (Sligte, Greer y De Dreu, 2012)

\section{Desarrollo}

La teoría institucional enmarca a la legitimidad como un factor clave para que una organización pueda alcanzar el éxito (Meyer \& Rowan, 1977). De otro modo, la legitimidad mejora las oportunidades para adquirir todos los recursos necesarios para la supervivencia y el crecimiento, como el capital, tecnología, equipos de gestión, personas, clientes y redes de trabajo (Meyer \& Rowan, 1977; Zucker, 1987; Aldrich \& Fiol, 1994; Scott, 1995); y su supervivencia en el tiempo se verá reflejada con el apoyo que la organización reciba por parte de sus grupos de interés (Arnold et al., 1996).

La legitimidad proporciona una base para la toma de decisiones diferente a otros medios racionales, de forma que las personas son influenciadas al creer que las decisiones tomadas por otras personas $\mathrm{u}$ organizaciones legitimadas son correctas o 
apropiadas y deben ser seguidas (Zelditch, 2001); además "los grupos de interés desean que las organizaciones se ajusten a determinadas normas de comportamiento socialmente aceptadas", aseguran (Díez, Blanco y Prado, 2010, p. 131).

La percepción generalizada o asunción de que las actividades de una entidad son deseables, correctas o apropiadas dentro de algún sistema socialmente construido de normas, valores, creencias y definiciones (Suchman, 1995). Así mismo, se afirma que la legitimidad consiste en un estado que refleja la alineación cultural, el apoyo normativo o la consonancia con las reglas y leyes relevantes (Scott, 1995); a su vez, la percepción de legitimidad o justicia dentro de la organización por parte de los empleados se relaciona positivamente con conductas positivas hacía la empresa, más allá incluso de sus obligaciones como trabajadores (Tziner y Sharoni, 2014). Por otro lado (Cruz, 2008) ha hecho su postulado en la idea de que la legitimidad se define según el reconocimiento que den varios sujetos, partiendo de una validación grupal pues esto soportará aún más el consentimiento sobre el buen actuar de una compañía.

Las certificaciones que acreditan a cualquier organización en el actuar reflejan una medición acorde con los estándares preestablecidos, y con la cual se determinaría una evaluación en la aplicación del marco normativo y el logro de los objetivos (Ruef y Scott, 1998).

Las organizaciones que deseen mantener una estabilidad a través del tiempo, deben tener en cuenta su entorno, puesto que la legitimidad tiene relación con la percepción de la manera como alcanzan los objetivos (Díez, Prado y Blanco, 2010). De la misma manera, estos autores afirman que entre mejor sea la percepción de la sociedad, mejores serán los resultados que se puedan obtener, y además es un instrumento del cual las empresas que hasta ahora llegan al mercado pueden tomar provecho para mejorar su posición y reconocimiento, pues las personas manejan una visión positiva del cómo actúa la organización, y esto tendrá una mejor aceptación y ayudara a conseguir los objetivos propuestos de manera más sencilla (Andrews, 1996; Zimmerman y Zeit, 2002; Rutherford \& Buller, 2007; Zsidisin, Melnyk y Ragatz, 2005). La legitimidad mejora la estabilidad y comprensibilidad de las actividades organizativas mostrando la inclusión de la organización en un sistema institucionalizado de creencias y acciones.

\section{Legitimidad Moral}

Scott (1977) y Scott y Meyer (1991) basaron sus definiciones en legitimidad moral, asegurando que para su identificación se examinarán los procedimientos y formas en que la organización se soporta para el logro de los objetivos, haciendo también necesaria una evaluación normativa positiva de la organización y sus actividades (Parsons, 1960; Aldrich \& Fiol, 1994). Una organización demuestra que es deseable desde una dimensión moral cuando el trato hacia los empleados o hacia los clientes es el esperado dentro del sistema social donde opera, o cuando se consigue los beneficios deseados para la actividad que se desarrolla.

\section{Legitimidad Cognitiva}

Este tipo de legitimidad se asocia con la percepción del desarrollo de las acciones de una organización, en la forma más apropiada; cuando sus grupos de interés consideran que el desarrollo de sus estrategias se cumplen de la mejor forma posible teniendo en cuenta el sistema, los procedimiento y los recursos (Thomas, 2005). La legitimidad cognitiva viene derivada de tomar como propio el sistema de creencias, formulado por profesionales y científicos, donde se especifica y codifica el conoci- 
miento, dando por sentado supuestos que aportan un marco de actuación para las rutinas diarias y también para las más especializadas (Scott citado por Díez, Prado y Blanco, 2010). Teniendo en cuenta que este tipo de legitimidad se fundamenta en el conocimiento (Aldrich \& Fiol, 1994), la organización debe mostrar que las actividades desempeñadas son aceptadas de manera positiva en el lugar en que funciona. (Aldrich \& Fiol, 1994; Scott, 1995; Suchman, 1995; Hunt \& Aldrich, 1996; Zimmermann y Zeit, 2002).

\section{Legitimidad Pragmática}

Apoyan las acciones de la organización, pues la consideran como una persona que demuestra y comparte sus intereses, valores, creencias y que es honesta, deseable, auténtica y fiable. Una organización muestra legitimidad pragmática cuando sus objetivos se encuentran en consonancia con los intereses de sus grupos de presión y se reconoce en una organización cuando ejecuta acciones dirigidas a la obtención de esos objetivos (Cruz-Suárez, Díez-Martín, Blanco-González y Prado-Román, 2014).

\section{Variables de Legitimidad}

- Valores: Los valores éticos son estructuras de pensamiento que se mantienen preconfiguradas de cara a una supervivencia como especie humana (García y Dolan, 1997). La disposición relativamente constante de hacer u obrar de forma determinada, constituye el hábito, este supone un acto deliberado, bueno (virtud) o malo (vicio). Ahora bien, la virtud proviene tanto del vocablo latino virtus vir y vis: varón y fuerza, como del griego areté: perfección, mérito o cualidad. En este estudio se consiente en definir la virtud como la capacidad y habilidad de efectuar acciones adecuadas a la persona humana según lo propone López (2000).
- Estructura organizacional: Ackoff (2000), Daft y Steers (1992), Galbraith, Downey \& Kates (2001), Litterer (1979), Mintzberg (1984), Nadler y Tushman (1997) afirman que las organizaciones establecen patrones de comportamiento para estandarizar los procesos y actividades que se desarrollen.

- Relaciones con el entorno: "La organización donde uno se sitúa resulta afectada positiva o negativamente por procesos y/o centros de poder y decisión más o menos ajenos al control de la organización” (Cruz, 1998, p .34).

- Grupos de interés: También denominado stakeholder es definido por Freeman (1984) como "aquellos grupos o individuos que pueden afectar o lo están por el logro de objetivos de la organización". El poder de los "grupos de interés" nace apoyado por la relevancia cada vez mayor de factores que lo respaldan e impulsan.

\section{Desempeño Organizacional}

A partir del Modelo de Bontis, se toma el rendimiento organizacional como un constructo social que puede ser percibido por los grupos de interés (Real, 2003; Cardona y Calderón, 2006) y precisamente se acogen las variables empleadas por estos últimos investigadores. Para tal efecto el rendimiento es un constructo social basado en la percepción del sujeto. Lo cual hasta el momento algunos autores han basado sus escritos en desempeño organizacional, y más exactamente en la medición de este en unas variables que permiten determinar la percepción de las personas que pertenecen a la organización.

Para esta investigación es de gran importancia los resultados que han encontrado otros investigadores, pues sirven como soporte de validez en cuanto al desarrollo de la medición del desempeño organizacional. 
Para la medición del desempeño organizacional se fundamentó en la percepción de los individuos de las organizaciones, que según autores como Suñé (2004), Bontis, Crossan y Hulland (2000), se proponen algunos elementos para dicha medición. Esta investigación dejará de lado el rendimiento económico de la organización y se basará tan solo en el desempeño organizacional medido a partir de la opinión del sujeto.

El desempeño organizacional según Bontis et al (2000) lo definen como un constructo haciendo la medición a través de la percepción; así mismo Suñé (2004) plantea la evaluación del desempeño a partir de la opinión del sujeto. En el modelo de Bontis et al. (2000) se encuentran cinco variables para la medición del rendimiento de las organizaciones desde la opinión de las personas:

- Nuestra organización es exitosa.

- El grupo reúne sus objetivos de rendimiento.

- Las personas están felices de trabajar aquí.

- Nuestra organización cumple con las necesidades de sus clientes.

- El desempeño futuro de nuestra organización es seguro.

Mientras Suñé (2004) plantea otras cinco variables para la evaluación del rendimiento de la organización teniendo en cuenta también la percepción de las personas de la organización:

- Los objetivos de la organización son alcanzados plenamente.

- Los recursos de nuestra organización se aprovechan eficazmente.

- Nuestra organización evoluciona más rápidamente que sus competidores.

- Nuestra organización es un ejemplo de buena coordinación.

- Nuestra organización es capaz de reinventarse a sí misma.
Cardona y Calderón (2006) en "El impacto del aprendizaje en el rendimiento de las organizaciones", proponen dos variables más:

- La organización es respetada.

- Nuestra organización reacciona con rapidez a los cambios del entorno y toma decisiones proactivas e informadas.

Estas variables junto con las de la legitimidad permiten establecer la asociación que se demuestra empíricamente.

De otro modo, la legitimidad mejora las oportunidades para adquirir todos los recursos necesitados para la supervivencia y el crecimiento, como el capital, tecnología, equipos de gestión, personas, clientes y redes de trabajo (Meyer \& Rowan, 1977; Zucker, 1987; Aldrich \& Fiol, 1994; Scott, 1995). Esto viene motivado como consecuencia de que los grupos de interés desean que las organizaciones se ajusten a determinadas normas de comportamiento socialmente aceptadas, aseguran Diez, Blanco y Prado (2010). Además, la legitimidad proporciona una base para la toma de decisiones diferente a otros medios racionales, de forma que las personas son influenciadas porque creen que las decisiones tomadas por otras personas $u$ organizaciones legitimadas son correctas o apropiadas y deben ser seguidas (Zelditch, 2001).

\section{Metodología}

Con una investigación de tipo cualitativo se realizó un estudio exploratorio y descriptivo, con el fin de identificar las conductas de la Escuela de Administración de Empresas en la facultad con Sede en la seccional Sogamoso de la Universidad Pedagógica y Tecnológica de Colombia, bajo un método inductivo el cual permite interactuar constantemente a los investigador y los datos, además de permitir 
generalizar a partir de sus observaciones, y tiene como objetivo generar nuevo conocimiento, direccionando de lo particular a lo general. Las técnicas y herramientas de recolección de información que permitieron reconocer la percepción de los diferentes actores son la observación y la entrevista, las cuales permitieron obtener datos relevantes para el logro de los objetivos planteados en la investigación.

Al realizar entrevistas a los diferentes grupos de interés con el fin de conocer la percepción que tienen acerca de la Escuela de Administración de Empresas, de la facultad con Sede en la Seccional Sogamoso de la Universidad Pedagógica y Tecnológica de Colombia, las preguntas salieron de las variables encontradas en el marco teórico y que afectaron tanto la legitimidad organizacional como el desempeño organizacional que además concluye con un análisis documental.

Sin embargo, los estudios realizados sobre legitimidad aseguran que por la dificultad de su medición se deben desarrollar de manera mas teórica que empírica (Bozeman, 1993; Suchman, 1995; Low \& Johnston, 2008). No obstante, vale la pena resaltar el trabajo de Deephouse (1996), quien plantea que es posible medir la legitimidad examinando las evaluaciones a nivel de aceptación de los actores sociales, tanto el gobierno como el público en general. Por tal razón, en esta investigación al igual que en la elaborada por Deephouse (1996) se tienen como sujetos a los stakeholder, incluyendo a un representante del estado, que en este caso fue por parte de la alcaldía; y de otro lado, dentro del concepto de público en general se incluyó a los usuarios (estudiantes), directivas de la universidad, empleados, empresarios, egresados, competencia y medios de comunicación.

A partir de la reflexión teórica realizada, surgen las preguntas para la entrevista estructurada que se aplicaba, don- de se buscaba reconocer la percepción de los stakeholder, a través de interrogantes como: ¿conoce al Programa de Administración de Empresas?, ¿qué noticias ha escuchado sobre la actividad que realiza?, itiene conocimiento de aspectos negativos del programa?, ¿cuál es la relación del programa con el estado y las empresas de la región?, ¿los egresados son visualizados y reconocidos en el ambiente laboral?, ¿frente a la competencia, cómo se encuentra el programa?, ¿desarrolla el programa actividades de investigación, extensión y proyección social?, entre otras preguntas que permitieron establecer y describir las características propias del programa frente a legitimidad y su desempeño organizacional.

\section{Resultados}

Tras la realización de las entrevistas, se denota que los diferentes sujetos tienen muchas afirmaciones en común, permitiendo deducir que el desempeño de la Escuela de Administración de Empresas de la Universidad Pedagógica y Tecnológica de Colombia, sede Seccional Sogamoso se deriva de las actividades que esta realiza a diario y estas son influenciadas por su legitimidad, es decir el programa viene desarrollando acciones que son aceptadas y deseadas por la sociedad. Lo anteriormente dicho se puede soportar, no solo por las entrevistas realizadas, sino también por el análisis documental, que revela como la escuela se ciñe bajo los parámetros de la universidad en general, pero también a través de los años los registros calificados que se han recibido y garantizan y aseguran a toda la comunidad que es una institución aceptada por el Ministerio de Educación según la legislatura del país. No todos los programas de educación superior alcanzan a obtener el registro calificado, haciendo de esto uno de los principales indicadores para cualquier institución. 
La Escuela de Administración de Empresas tiene una estructura definida, un plan de acción que garantiza tener certeza del qué, el cómo y el cuándo se deben realizar cada una de las actividades en pro de alcanzar los objetivos planteados para cada período, y también certifica que la toma de decisiones va soportada en el buen actuar del programa asociando dichas variables a algunas del desempeño como el hecho de que la organización es coordinada, respetuosa y exitosa.

En cuanto a las variables de relaciones con el entorno, visión de especialistas, valores y evaluación normativa, enfocadas más directamente a la legitimidad moral y cognitiva, se puede decir que tienen intima asociación con el desempeño que la escuela tiene, puesto que se encontró que los diferentes grupos de interés están satisfechos con el actuar del programa, y con las recomendaciones recibidas en el instrumento aplicado, las cuales son para mejorar y seguir creciendo como institución formadora de profesionales íntegros. También es importante recalcar que uno de los indicadores que la investigación tuvo en cuenta para la medición del desempeño organizacional fue la identidad, la cual tuvo asociación precisamente con variables como los valores, la evaluación normativa y el buen nombre, provocando tanto en estudiantes como docentes y demás trabajadores orgullo y sentido de pertenencia hacia la institución.

Una de las variables más claras y en ocasiones obvia para determinar el reconocimiento de una organización es el "Buen Nombre", y de manera positiva en el estudio realizado, se concluye como la Escuela no ha sido afectada por "malas noticias" en prensa o radio, así como tampoco ha recibido sanciones por actuaciones corruptas, de fraude o que atenten contra el bienestar de algún grupo de interés, sino por el contrario es bien reconocida y su nombre testifica y es calificativo de una institución honorable y respetada; razón por lo cual muchas personas con deseos de ingresar a la educación superior tienen como primera opción al Programa de Administración de Empresas de la Universidad Pedagógica y Tecnológica de Colombia -UPTC- y en particular en la Sede Sogamoso. En la teoría se afirma que el buen nombre de una organización está íntimamente asociado al éxito, liderazgo, respeto y satisfacción de clientes internos y externos y dicha asociación se comprueba en esta investigación asegurando con plenitud que el buen desempeño de la Escuela es procedente de dicha variable de legitimidad.

\section{Conclusiones}

La legitimidad es un tema que ha fortalecido su estudio a través de investigaciones debido a su importancia para cualquier tipo de organización y en efecto se debe tener en cuenta la percepción de los individuos que están relacionados a la sociedad y lugar donde se opera.

En la revisión bibliográfica se sustenta la asociación existente entre legitimidad organizacional y el desempeño organizacional, encontrando como la legitimidad es un factor importante para las organizaciones por ser garantia de la supervivencia y estabilidad en el tiempo.

En la revisión de literatura realizada se pudo apreciar como en Colombia son escasas las investigaciones sobre este tema, a pesar de ser parte esencial para cualquier tipo de organización, por lo cual la literatura consultada y trabajada en su gran mayoría se encontraba en el idioma inglés.

Los entrevistados con algún tipo de relación directa con la Escuela aseguran que los objetivos se están alcanzando a plenitud, y la imagen en la sociedad inmediata es buena, por la ausencia de casos de corrupción o "malas prácticas". Además, 
por escrito o de manera sustentable tampoco existen casos que demuestren "malas relaciones" entre estudiantes, docentes o directivos con la escuela, llegando a deducir que moralmente la Escuela es legítima.

Como estudiante de la Escuela de Administración de Empresas es un honor haber realizado una investigación sobre el comportamiento de la misma, por el mismo deseo académico y de alguna manera servir como soporte para procesos en pro de su mejora; encontrando como a pesar de las diferentes perspectivas sobre la Escuela se logran alcanzar sus objetivos de una manera deseable y aceptada por la sociedad.

\section{Referencias}

Ackoff, R. (2000). Recreación de las corporaciones: un diseño organizacional para el siglo XXI. México DF: Oxford University Press.

Alcantara, L., Mitsuhashi, H. y Hoshino, Y. (2006). Legitimacy in international joint ventures: It is still needed. Journal of International Management, 12(4), 389-407. https://doi.org/10. 1016/j.intman.2006.08.002

Aldrich, H. \& Fiol, C. (1994). Fools rush in? The institutional context of industry creation. Academy of Management Review, 19(4). 645-670. https://doi.org/ $10.2307 / 258740$

Andrews, A. (1996). Signaling legitimacy after metamorphic change: Directors in the initial public offering. Congreso Anual de la Academy of Management, Cincinnati, USA.

Arnold, S., Handelman, J. y Tigert, D. (1996). Organizational legitimacy and retail store patronage. Journal of $\mathrm{Bu}$ siness Research, 35(3). 229-239. https://doi.org/10.1016/0148-2963(95) 00128-X
Ashforth, B. \& Gibbs, B. (1990). The double-edge of organizational legitimization. Organization Science, 1(2). 177-194. https://doi.org/10.1287/orsc.1. 2.177

Bontis, N., Crossan, M. \& Hulland, J. (2002). Managing an organizational learning system by aligning stocks and flows. Journal of Management Studies, 39(4). 437-469. https://doi.org/10.1111/ 1467-6486.t01-1-00299

Bansal, P., y Clelland, I. (2004). Talking trash: Legitimacy, impression management, and unsystematic risk in the context of the natural environment. Academy of Management Journal, 43(4), 717-736. https://doi.org/10.5465/ 20159562

Bozeman, B. (1993). Understanding the roots of publicness. En B. Sutton (Ed). The legitimate corporation. (63-81). Cambridge: Blackwell.

Cardona, J. y Calderón, G. (2006). El impacto del aprendizaje en el rendimiento de las organizaciones. Cuadernos de Administración, 19(32). 11-43.

Cruz-Suaréz, A,, Díez-Martín, M., BlancoGonzález, A. y Prado-Román, C. (2014). Análisis de las relaciones entre la legitimidad organizativa, sus fuentes y dimensiones. Revista Venezolana de Gerencia. 19(65). 9-22. Recuperado de http://www. redalyc.org/pdf/290/29030101002.pdf

Cruz, F. (1998). "Sobre el Entorno Cultural”. En Entorno Organizacional, Guzmán, A. J. (Compilador). Cali, Colombia: Facultad de Ciencias de la Administración, Univalle.

Cruz, L. (2008). El concepto de legitimidad en la autoridad: elementos de análisis para comprender la relación autoridadsubordinación en el comportamiento administrativo. Academia, 40(1). 6882. Recuperado de http://www.redalyc. org/articulo.oa?id=71612100006 
Daft, R. y Steers, R. (1992). Organizaciones: el comportamiento del individuo $y$ de los grupos humanos. México, D.F.: Limusa.

Deephouse, D. (1996). Does isomorphism legitimate? Academy of Management Journal, 39(4). 1024-1039. https://doi. org/10.5465/256722

Deephouse, D. y Suchman, M. (2008). Legitimacy in Organizational Institutionalism. In, R. Greenwood, C. Oliver, R. Suddaby \& K. Sahlin (Ed.), The SAGE Handbook of Organizational Institutionalism. (49-77). Newbury Park: Sage. https://doi.org/10.4135/9781849200387. n2

Díez, M., Blanco, A. y Prado, C. (2010). Legitimidad como factor clave del éxito organizativo. Investigaciones Europeas de Dirección y Economía de la Empresa, 16(3). 127-143. https://doi.org/10.1016/ S1135-2523(12)60038-0

Dimaggio, P. \& Powell, W. (1983). The iron cage revisited: Institutional isomorphism and collective rationality in organizational fields. American Sociological Review, 48(2). 147-160. https://doi.org/ $10.2307 / 2095101$

Dowling, J. \& Pfeffer, J. (1975). Organizational legitimacy: Social values and organizational behaviour. Pacific Sociological Review, 18(1). 122-136. https://doi.org/ $10.2307 / 1388226$

Freeman, E. (1984). Strategic Management: A Stakeholder Approach, Boston: Printman Press.

Fombrun, C. \& Shanley, M. (1990). "What's in a Name? Reputation Building and Corporate Strategy. Academy of Management Journal, 33(2). 233-258. https:// doi.org/10.5465/256324

Galbraith, J. Downey, D. \& Kates, A. (2001). Designing dynamic organizations, a hands-on for leaders at all levels. New York: Amacom.
García, S. y Dolan, S. (1997). La Dirección por Valores: el cambio más allá de laDirección por Objetivos. Madrid: McGrawHill-IESE.

Human, S. \& Provan, K. (2000). Legitimacy building in the evolution of small firm mutilateral networks: acomparative study of success and demise. Administrative Science Quarterly, 45(2). 327365. https://doi.org/ 10.2307/2667074

Hunt, C. \& Aldrich, H. (1996). Why even Rodney Dangerfield has a home page: Legitimizing the world wide web as a medium for commercial endeavours. Congreso Anual de la Academy of Management, Cincinnati, USA.

Jun, L., Qiuzhen, W. \& Qingguo, M. (2011). The effects of project un certa in tyan drisk management on IS development project performance: A vendor perspective. International Journal of Project Management, 29(1). 923-933. https:// doi.org/10.1016/j.ijproman.2010.11.002

Lammers, J., Galinsky, A. D., Gordijn, E. H. y Otten, S. (2008). Illegitimacy moderates the effects of power on approach. Psychological Science, 19(1), 558-564. http://dx.doi.org/10.1111/j.14679280.2008.02123.x

Litterer, J. (1979). Análisis de las organizaciones. México DF: Limusa

López, A. (2000). Valores, Valoraciones Y Virtudes. México, D.F.: CECSA.

Low, B. \& Johnston, W. (2008). Securing And Managing An Organization's Network Legitimacy: The Case of Motorola China. Industrial Marketing Management, 37(7). 873-879. https://doi.org/10. 1016/j.indmarman.2008.01.009

Meyer, J. \& Rowan, B. (1977). Institutionalized organizations: Formal structures as myth and ceremony. American Journal of Sociology, 83(2). 340-363.

Mintzberg, H. (1984). La estructuración de las organizaciones. Barcelona: Ariel. 
Nadler, D. y Tushman, M. (1997). El diseño de la organización como arma competitiva: el poder de la arquitectura organizacional. México, D.F.: Oxford University Press

Parsons, T. (1960). Structure and process in modern societies. Glencoe: Free Press.

Real, J. (2003). Aprendizaje organizativo y creación de conocimiento en el desarrollo de competencias distintivas tecnológicas. [Tesis doctoral no publicada]. Universidad Pablo de Olavide, Sevilla, España

Reimann, F., Ehrgott, M., Kaufmann, L. \& Carter, C. (2011). Local stakeholders and local legitimacy: MNEs' social strategies in emerging economies. Journal International Management, 18(1). 1-17. http://dx.doi.org/ 10.1016/j.intman.2011. 06.002

Ruef, M. \& Scott, R. (1998). A multidimensional model of organizational legitimacy: Hospital survival in changing institutional environments. Administrative Science Quarterly, 43(4). 877-904. https://doi.org/10.2307/2393619

Rutherford, M. \& Buller, P. (2007). Searching for the legitimacy threshold. Journal of Management Inquiry, 16(1). $78-92$. h t t p s : / / d o i. org/10.1177/1056492606297546

Scott, W. (1995). Institutions and organizations. Thousand Oaks: Sage.

Scott, W. (1994). Conceptualizing organizational fields: Linking organizations and societal systems. In, H. Derien (ed.), Systemrationalitat und Partialinteresse. (203-221). Baden-Baden: Nomos Verlag.

Scott, W. (1977). Some problems in the study of organization structure. Mid-American Review of Sociology, 2(1). 1-16. https://doi.org/10.17161/STR.1808.4801
Scott, W. \& Meyer, J. (1991). The organization o societal sectors. En W. Powell y P. DiMaggio (Eds.) The new institutionalism in organizational analysis. (108-140). Chicago: University of Chicago Press.

Sligte, D., Greer, L. y De Dreu, C. (2012). Illegitimacy undermines lea-der creativity only when stable power.Revista de Psicología Social,27(1), 347-354. h t t p://dx.doi.org/10.1174/ 021347412802845603

Suchman, M. (1995). Managing Legitimacy: Strategic and Institutional Approaches. Academy of Management Review, 20(1), 571-611. https://doi.org/10.2307/ 258788

Suñé, A. (2004). El impacto de las barreras de aprendizaje en el rendimiento de las organizaciones. [Tesis doctoral no publicada]. Cataluña: Universidad Politécnica de Cataluña.

Thomas, T. (2005). Are business students buying it? A theoretical framework for measuring attitudes toward the legitimacy of environmental sustainability. Business Strategy and the Environment, 14(3). 186-197. https://doi.org/10.1002/ bse. 446

Tornikoski, E. \& Newbert, S. (2007). Exploring the Determinants of Organizational Development: A Legitimacy Perspective. Journal of Business Venturing, 22(1), 311-335. https://doi.org/10. 1016/j.jbusvent.2005.12.003

Tziner, A. y Sharoni, G. (2014). Organizational citizenship behavior, organizationaljustice, job stress, and workfamily conï $\neg$,ict: Examination of their interrela-tionships with respondents from a non-Western culture. Revista de Psicologíadel Trabajo y de las Organizaciones, 30(1), 35-42. http://dx.doi.org/10. $5093 / \operatorname{tr} 2014 a 5$ 
Willis, G., Guinote, A. y Rodríguez-Bailón, R. (2010). Illegitimacy improves goalpursuit in powerless individuals. Journal of Experimental Social Psychology, 46(1), 416-419. http://dx.doi.org/10.1016/ j.jesp.2009.10.009

Willis, G. y Rodríguez-Bailón, R. (2011). Si merezco tener poder, ¿qué más puedo esperar?: Poder, Legitimidad y Optimismo. International Journal of Social Psychology, 26(2). 241-255- https://doi. org/10.1174/021347411795449018

Zelditch, M. (2001). Processes of legitimation: recent developments and new directions. Social Psychology Quarterly. 64(1). 4-17. http://dx.doi.org/10.2307/ 3090147

Zsidisin, G., Melnyk, S. \& Ragatz, G. (2005). An institutional theory perspective of business continuity planning for purchasing and supply management. International Journal of Production Research, 43(16), 3401-3420. https://doi. org/10.1080/00207540500095613

Zimmerman, M. \& Zeitz, G. (2002). Beyond survival: achieving new venture growth by building legitimacy. Academy of $\mathrm{Ma}$ nagement Review, 27(3). 414-31. https:// doi.org/10.5465/amr.2002.7389921

Zucker, L. (1987). Institutional theories of organization. Annual review of Sociology, 13(1). 443-464. http://dx.doi.org/10. 1146/annurev.so.13.080187.002303
Dora Esther Fonseca Pinto es Magíster en Administración Económica y Financiera de la Universidad Tecnológica de Pereira (Colombia) y Administradora de Empresas, docente investigadora de la Universidad Pedagógica y Tecnológica de Colombia-UPTC-(Colombia).

Jenny Mairena Herrera Rodríguez es estudiante de Maestría en Seguridad y Salud en el Trabajo con Énfasis en Higiene y Seguridad Industrial y Administradora de Empresas de la UPTC, e investigadora de la misma institución. 